\title{
Note sur une nouvelle station d'Asplenium sagittatum (DC.) Bange dans la région de Tlemcen (Algérie occidentale)
}

\section{Brahim Babali', Boumediene Medjahdi² \& Mohammed Bouazza ${ }^{1}$}

${ }^{1}$ Laboratoire d'écologie et gestion des écosystèmes naturels, département d'Ecologie et environnement. Université de Tlemcen

'Laboratoire de Gestion Conservatoire de l'eau, du Sol et des Forêts et Développement durable des zones montagneuses de la région de Tlemcen. Université de Tlemcen.

\section{Correspondencia}

B. Babali

e-mail: miharb_babali@hotmail.fr

Recibido: 13 noviembre 2017

Aceptado: 19 diciembre 2017

Publicado on-line: diciembre 2017
Note on a new station of Asplenium sagittatum (DC.) Bange Bange in Tlemcen'sarea (Western Algeria).

Mots clés: Aspleniaceae, Asplenium sagittatum, répartition, espèce rare, Tlemcen.

Key words: Aspleniaceae, Asplenium sagittatum, distribution, rare species, Tlemcen.
Les ptéridophytes sont les seuls cryptogames vasculaires, groupe intermédiaire entre les phanérogames et les autres plantes inférieures (Prelli, 2001). Le nombre de taxons de Ptéridophytes acceptés en Afrique du Nord est de 114 ; dont 58 taxons sont présents en Algérie (Dobignard et Chatelain, 2010) sur les 58 taxons, 26 ont été signalés dans la région de Tlemcen. (Medjahdi et al., 2013)
Dans ce travail nous avons découvert une nouvelle station d'Asplenium sagittatum. Cette espèce de distribution circumméditerranéen est assez rare en Algérie. II a été cité par plusieurs auteurs (Munby 1858 ; Battandier et Trabut1902, Maire 1952, Quézel et Santa 1962, Medjahdi et al., 2013,) dans le centre et l'Ouest de l'Algérie dans les secteurs biogéographiquessuivantes: Kabylie (K I-2-3), Algérois (Al), Oranie (O3) : Ghar-Rouban.

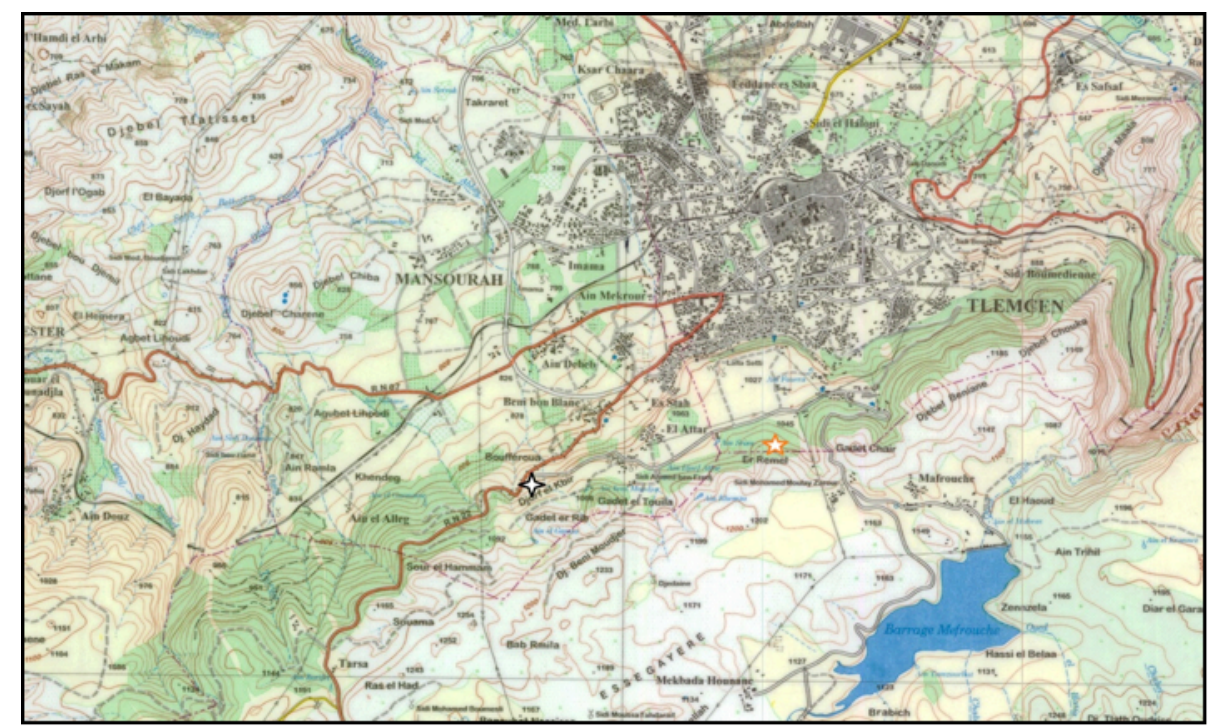

Figure 1. Stations nouvelles d'Asplenium segetatum dans la région de Tlemcen. (Etoile rouge à 5 branches : station de Doumergue 1872 ; Etoile noir à 4 branches : notre observation 2016) 


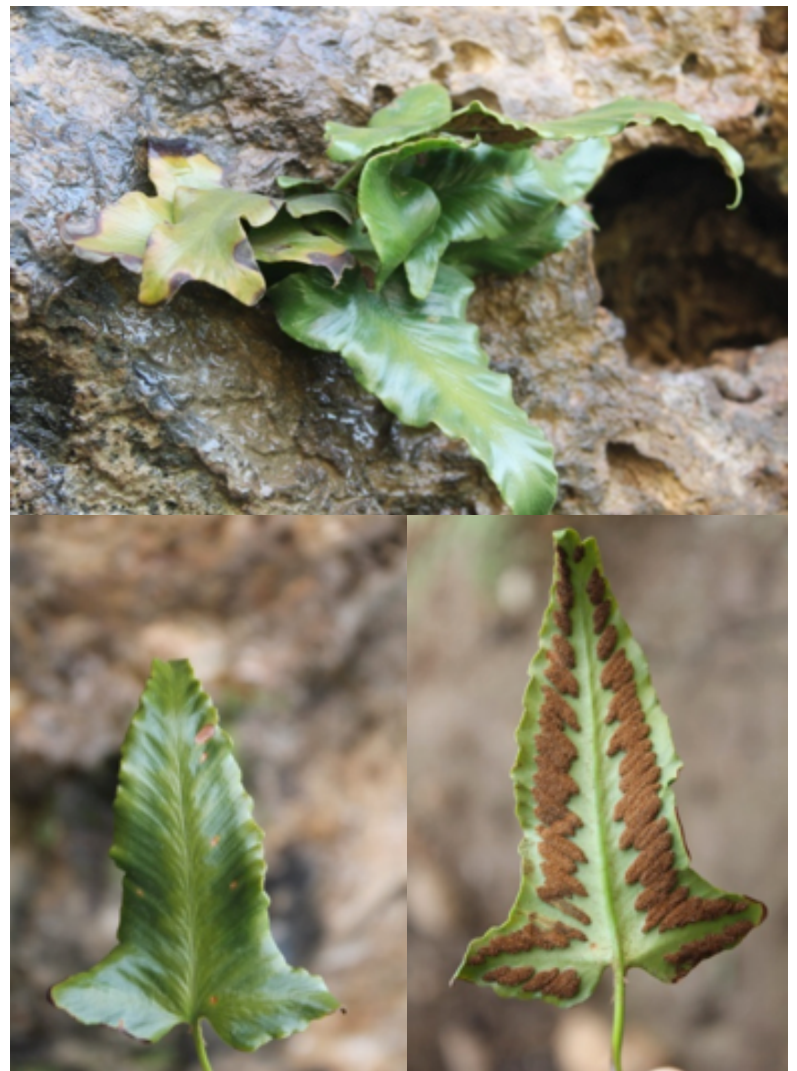

Figure 2. Asplenium segetatum(DC.) Bange. (À gauche vue générale ; au milieu face supérieur ; à droite face inferieur de la fronde)

Même si l'espèce est présente dans plusieurs stations du centre et l'Est dans les régions plus ou moins humides. En Oranie cette ptéridophyte n'est présente que dans la région de Tlemcen.

Dans cette région, l'Asplenium sagittatuma été signalé par Pomel (1874) dans les rochers humides ombragés à Ghar-Rouban. Une autre observation de Doumergue (in Santa, 1949) a été faite dans l'excavation au pied du Dj. Attar (1872 date proposée!), cette dernière station n'a pas été retrouvée donc n'a pas été citée par Maire (1952) ni Quézel et Santa (1962).

Nos prospections nous ont permis, non seulement de redécouvrir l'ancienne station, mais également dien découvrir une nouvelle station d'Asplenium sagittatum. En effet, l'ancienne station de Domergue est située dans la forêt de Petit Perdreaux à $1100 \mathrm{~m}$ altitude, sur la route du siège du Parc National de Tlemcen à quelque mètre du rond-point de cette route (au point GPS 34.860066, -1.320138). L'Asplenium sagittatum est fixé sur les roches calcaires dans la lisière de la forêt de Pinus halepensis Mill.

La nouvelle est située à $3 \mathrm{~km}$ à l'Ouest de la première sur la route de Tlemcen-Terni (lieu-dit col de Zariffet) au Nord-Ouest de la ville de Tlemcen au point GPS 34.852406, -1.348344 à 1030 m (fig. 1).

Nous avons trouvé 1 seul individu en bonne santé le 11 avril 2016 sur des roches calcaires humides. L'espèce s'associée avec une végétation plus ou moins denses : Asplenium ceterach L., Aristolochia baeticaL.,Aristolochia paucinervis Pomel, Poa bulbosa L., Lysimachia linum-stellatum L., Hyoseri sradiata L., Geranium atlanticum Boiss., Rupicapnos africana subsp. oranensis (Pugsley) Maire, Catananche caeruleav ar. Tlemcenensis Faure....

\section{Références}

Battandier et Trabut (1902). Flore analytique et synoptique de l'Algérie et de la Tunisie.460P

Dobignard, A. \& Chatelain, C. (2010). Index synonymique flore d'Afrique du Nord. Volume 1 : Ptéridophyta, Gymnospermae et Monocotyledoneae. Editions Des Conservatoire et Jardin Botaniques, Genève. 455 p.

Maire, R. (1952). Flore de l'Afrique du Nord. Volume 1. Ed. Le chevalier Paris.

Medjahdi, B., Letreuch-Belarouci, A., Prelly, R. (2013)Actualisation du catalogue des ptéridophytes du Nord-ouest algérien (Région de Tlemcen). Acta Bot. Malacitana, 38, 33-39

Prelli, R. (2001). Les fougères et plantes alliées de France et d'Europe occidentale

Quézel, P. \& Santa, S. (1962). Nouvelle flore de l'Algérie et des régions désertiques méridionales. CNRS, Paris, Tome 1. $558 \mathrm{p}$.

Santa, S. -1949 - Catalogue des plantes de l'Algérie occidentale et du Maroc oriental. Extrait de B.S.G.A. de la Provence d'Oran. T.72, Fascicule 226, 128p. 